\title{
In-Situ Analytical Study Of Bricks Exposed To Marine Environment Using Hand-Held X-Ray Fluorescence Spectrometry And Related Laboratory Techniques
}

Morillas, Héctor; García Florentino, Cristina; Marcaida, Iker; Maguregui, Maite; Gorka, Arana; Silva Oliveira, Luis Felipe; Madariaga, Juan Manuel.

\begin{abstract}
In this work, the degradation processes that take place in bricks exposed to marine environments have been studied. Taking into account the importance of this building material where the silicates present in the final product act as stabilizer in the porous material itself, it is necessary to understand the decay processes that occur in these aggressive environments. As is known, the marine aerosol carries different types of salts, such as chlorides, sulfates, nitrates, etc., present in surrounding environment exerting a negative influence on the materials producing cracking and disintegration processes of the material and consequently loss of brick wall stability. Nowadays the development of portable devices is taking much more importance helping researchers to resolve problems in the field in a fast and easy way. In order to extract fast and satisfactory results about the conservation state of different bricks from Punta Begoña Galleries (Getxo, Basque Country, Spain), an in-situ analytical methodology was developed based on the use of handheld Energy Dispersive X-ray fluorescence spectrometry (HH-ED-XRF) assisted with other laboratory techniques ( $\mu$-ED-XRF and X-Ray Diffraction) in order to corroborate and complement the information obtained in-situ. This construction undergoes the influence of marine aerosol, industrial port, power generation plants, and a fuel refinery among others. The pathologies visually observable in these bricks are disintegration, breakup and detachment of the bricks. The presence of deterioration compounds in the bricks has been studied according to the orientations of the bricks inside the construction.
\end{abstract}

\section{Keywords}

Brick; Calcium Silicate; HH-ED-XRF; In-Situ Analysis; Marine Environment 